\title{
Quantum mechanical investigation of the nature of nucleobase-urea stacking interaction, a crucial driving force in RNA unfolding in aqueous urea
}

\author{
NITISH ALODIA, TANASHREE JAGANADE and U DEVA PRIYAKUMAR* \\ Center for Computational Natural Sciences and Bioinformatics, International Institute of Information \\ Technology, Hyderabad, Telangana 500 032, India \\ E-mail: deva@iiit.ac.in
}

MS received 2 August 2018; revised 22 September 2018; accepted 24 September 2018; published online 1 November 2018

\begin{abstract}
Urea-assisted denaturation of protein and RNA has been shown to be a valuable tool to study their stabilities and folding phenomena. It has been shown that stacking interactions between nucleobases and urea are one of the driving forces of denaturation. In this study, the ability of urea to form unconventional stacking interactions with RNA bases is investigated by performing high-level quantum calculations (RI-MP2/aug-ccpVDZ level) on a few thousands of model systems. Four systems were considered based on the RNA nucleobases (GUA, ADE, CYT, and URA) for the investigation. For each system, a set of models were designed to study the role of hetero-atoms/groups of the nucleobases on stacking interactions with urea moiety with respect to every possible pair. Several plane-parallel complexes were generated with urea on top of aromatic systems to exhaustively study all possible factors for urea-nucleobases stacking interactions. Energy decomposition analysis (EDA), atoms in molecules (AIM) and natural bond orbital (NBO) analysis were performed to gain better insights on non-covalent stacking interactions. Dispersion component was found to be heavily stabilizing, while the $\mathrm{E}_{\mathrm{HF}}$ was found to be repulsive for all the four systems indicating lack of hydrogen bonding (HB) type interactions and presence of dispersion type interactions. Amide and carbonyl groups of urea molecule were found to play a major role in favourable stacking interactions. We demonstrate that along with functional groups present on the nucleobases, the orientation of urea molecules plays a vital role in stabilizing the urea-nucleobase non-covalent interactions. The proposed study quantifies and provides a comprehensive theoretical description of urea nucleobase unconventional stacking interactions which helps to unravel urea driven RNA unfolding mechanism.
\end{abstract}

Keywords. RNA unfolding; chemical denaturation; stacking interactions; substituent effect; dispersion interactions.

\section{Introduction}

During the last two decades, several experimental studies have unravelled crucial roles of RNA molecules in functional aspects in diverse cellular processes. ${ }^{1-3}$ Hence, a comprehensive understanding of structurestability-function relationships of RNA molecules is crucial. Osmolytes such as urea are known as osmoprotective partner due to its major role in protein denaturation and enzyme activity regulation and hence holds immense importance in living systems. ${ }^{4-7}$ Urea has attracted wide attention due to its denaturant

\footnotetext{
*For correspondence

properties which make urea an excellent chemical agent and the most common denaturant for protein unfolding and nucleic acid denaturation. ${ }^{8-16}$ Urea has been found to interact with polar as well as non-polar components of biopolymers and it can destabilize the native conformations of proteins and nucleic acids. ${ }^{12,17-20} \mathrm{~A}$ number of studies on urea-assisted protein unfolding greatly enhanced the understanding of urea driven protein denaturation mechanism. ${ }^{13,17,18,21-25}$ Extensive research during the last few decades on urea driven protein unfolding proposed two mechanisms by which urea assists in protein unfolding viz., the direct and indirect mechanism. ${ }^{22,26,27}$ The 'direct interaction' model 
suggests that urea binds to the protein directly and makes the unfolded state more stable. ${ }^{13,28,29}$ The 'indirect' model proposes that urea induces changes in the solvent environment, which leads to the unfolding of the protein. ${ }^{26,27}$ Most of the studies on protein unfolding provide valuable insights on the type and nature of interactions and explains the above-mentioned mechanisms in detail. ${ }^{9,30-33}$ However, such a detailed understanding of the mechanism of urea-induced RNA unfolding is not available.

The kinetic mechanisms of the folding phenomena of protein and RNA can be explored by studying their urea dependent folding and unfolding rates owing to urea's ability to denature folded states, which in turn can help us understand the thermodynamic stability of RNA and proteins. ${ }^{16,34-37}$ Although, extensive studies have given us an insight into the denaturation of proteins by urea, the mechanism and type of interactions due to which destabilization of RNA by urea takes place is still under investigation. RNA has been shown to regulate a number of cellular processes like gene regulation, RNA processing, catalysis reactions, transcription and protein synthesis. ${ }^{1,2,38,39}$ The function of RNA is the outcome of its structural features and hence it is important to understand the folding of RNA as well as its structural impacts on its function. ${ }^{40-42}$ The mechanism by which RNA changes its conformations in the presence of osmolytes like urea has become quite an intriguing field of research. ${ }^{43,44}$ Few studies have tried to understand the unfolding of RNA structure in presence of urea and propose a model for denaturation of RNA using urea. ${ }^{43,45}$ Computational studies revealed that urea interacts with nucleic acid bases via stacking and hydrogen bonding and stabilizes the denatured state of the RNA. ${ }^{15}$ Several studies have shown that urea forms stronger interactions with an exposed surface area of nucleobases during RNA denaturation process and hence, urea being a common denaturant of protein has also been used as a reagent to probe RNA folding. ${ }^{19,45}$ RNA nucleobases are strongly solvated by urea but the mechanism behind this is still not clear. Recently we showed using quantum mechanical $(\mathrm{QM})$ calculations that urea nucleobase stacking interactions are dominated by dispersion. ${ }^{46}$ Even though urea does not have a significant electron cloud like a typical aromatic system, the possibility of strong $\pi$-stacking interactions between urea and nucleic acid bases is rather intriguing. Noncovalent interactions are central to many areas of chemistry, materials science, and molecular biology. ${ }^{47-50}$ Though noncovalent interactions are weaker compared to covalent interactions, they can influence a lot of processes when acting together and hence minuscule changes in these interactions can have huge impacts. ${ }^{51-53}$ Due to the difference in structures of protein and RNA, the mechanism by which urea denatures RNA can be quite different than the process of protein denaturation by urea and thus a deeper understanding of how urea interacts with RNA, especially via stacking, is essential for reliable interpretation of the experimental studies. Furthermore, it is important to unravel the denaturation mechanism of RNA in presence of urea to understand the RNA structure-function relationship. To study the nature of the interactions between urea and RNA nucleobases and rationalize the importance of functional groups present on nucleobases and urea, quantum chemical calculations were performed on a number of model systems. In the current work, we aim to investigate the importance of different heteroatoms/groups of the nucleobase on the stability of urea-nucleobase stacking interactions, and the nature of such interactions.

\section{Computational methods}

To study stacking interactions of urea with biologically important $\pi$-systems, four systems were considered based on the RNA nucleobases (GUA, ADE, CYT, URA) each having a set of models resembling the nucleobases in terms of the number of $\pi$-electrons. Models were generated in such a way that the functional groups and heteroatoms of the target nucleobase were considered as variables, hence GUA, ADE, CYT and URA has 4, 4, 3 and 2 variables (substituents) respectively (Figure S1, Supplementary Information). For example, GUA can be considered as an indole molecule where a skeletal $\mathrm{CH}$ in the five-membered ring is replaced by a $\mathrm{N}$ atom, $\mathrm{CH}-\mathrm{CH}$ group is replaced by a CO-NH group, a $\mathrm{H}$ atom is replaced by $\mathrm{NH}_{2}$ group and another $\mathrm{CH}$ in the six-membered replaced by a $\mathrm{N}$ atom to get the original guanine base. Starting from the simplest model having no variables/substituents, the heteroatoms and substituents were systematically introduced leading to the target nucleobase. This resulted in a total of 16 models for GUA, 16 for ADE, 8 for CYT and 3 for URA (Figure 1). This approach helped us to study the effect of heteroatoms and different substituents on stacking interactions with urea. Planar structures of urea and all the models were optimized at the RI-MP $2^{54}$ level, using the aug-cc-pVDZ basis sets using the GAMESS program. ${ }^{55}$ For each model in each system, several complexes were made from the optimized planar structures of the model and the urea molecule such that the only difference between them was the position and orientation of urea with respect to the chosen molecule (Figure 2). For example, each of the 16 models of GUA base has nine atoms on the ring and two on the ring centers (six and five-membered). For each of the 11 positions, urea was positioned in six different orientations leading to a total of 1056 complexes. The common structural features of all the initial geometries were such that urea and the base molecule (model) were planar to each other and the interplanar distance was $3.0 \AA$ A The interplanar distances between urea and nucleobases in the stationary 


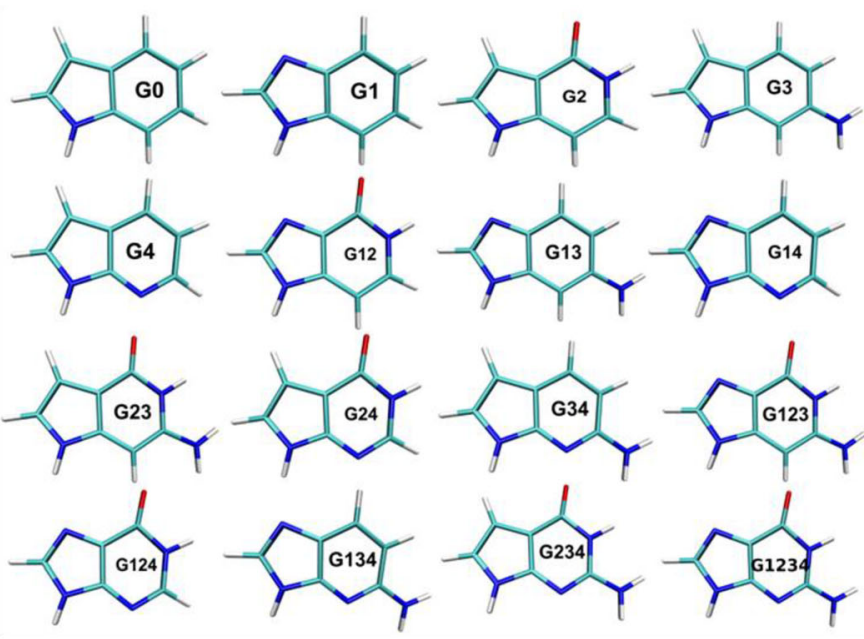

(a) Guanine models

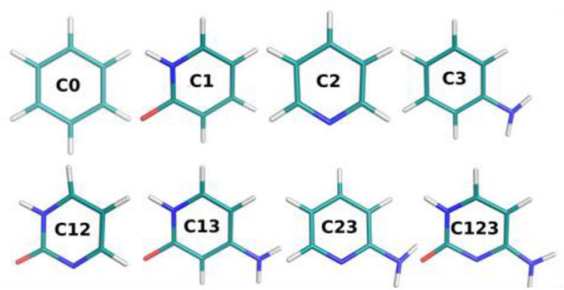

(c) Cytosine models
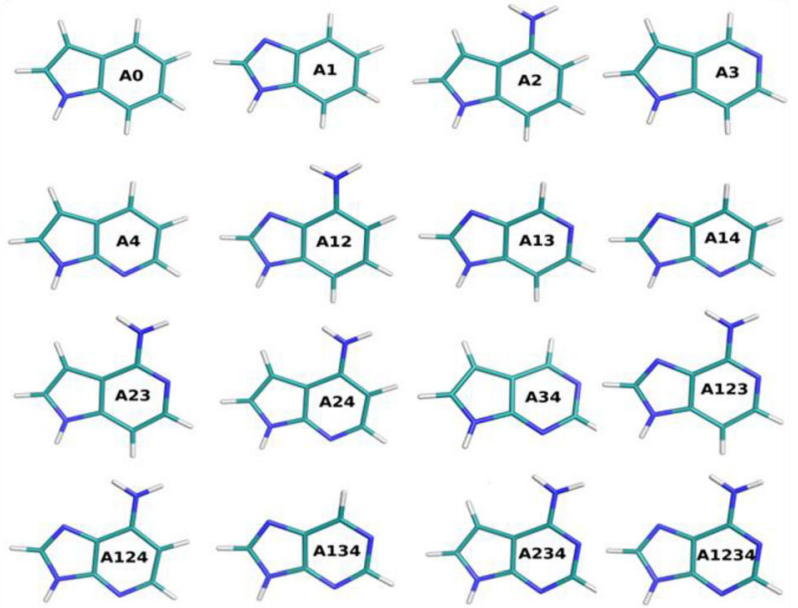

(b) Adenine models

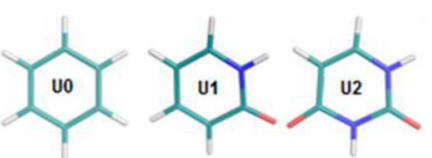

(d) Uracil models

Figure 1. Model system details considered in this study generated by using functional groups and heteroatoms as variables. (a) 16 Models of Guanine, (b) 16 Models of Adenine, (c) 8 Models of Cytosine, and (d) 3 Models of Uracil.
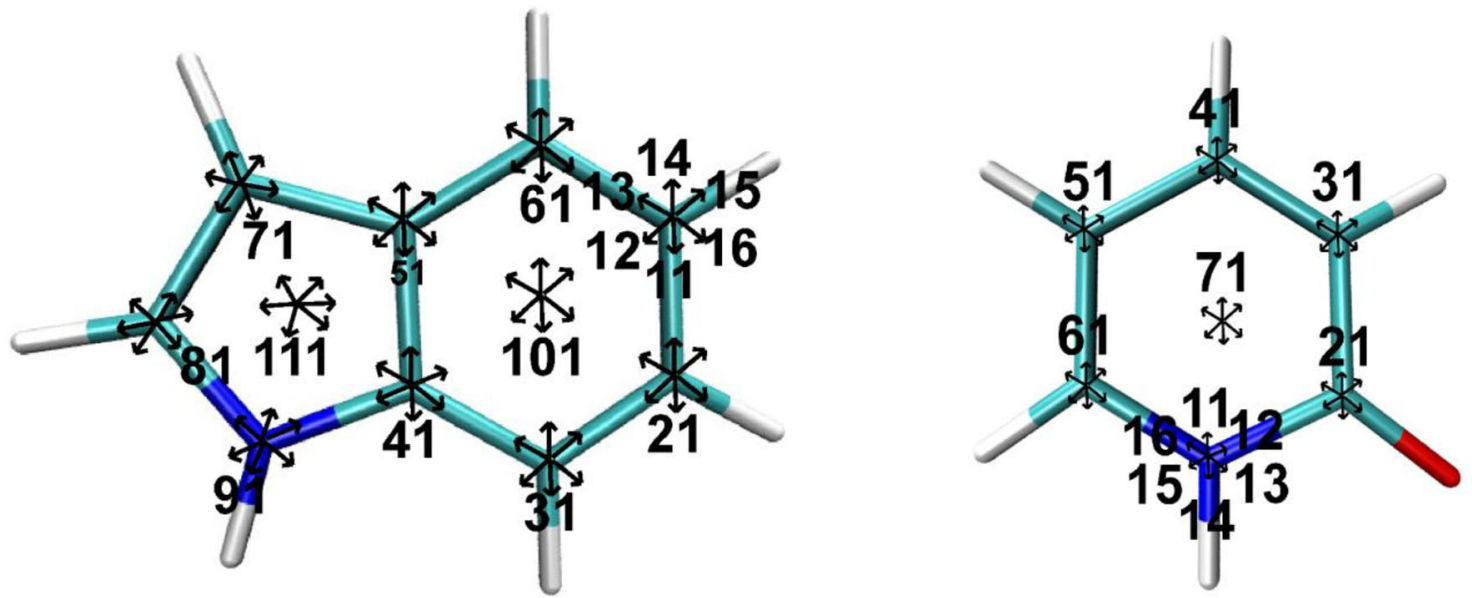

Figure 2. Numbering scheme used for each of the models of purines and pyrimidines with respect to the orientation of urea molecule and positions of the rings. Urea moiety placed in six different orientations with black arrows indicating the orientation of its carbonyl group on each of the 11 and 7 positions of purines and pyrimidines, respectively, which led to the formation of a total of 66 (two-membered ring models) and 42 (single membered ring models) complexes for each of the models generated in Figure 1 (in some cases, complexes are less than 66 and 42 due to symmetry).

points obtained using the optimization of nucleobase-urea binary complexes in the stacking arrangement was found to be close to $3 \AA{ }^{46}$ Hence, a total of 1056 complexes for GUA, 1056 for ADE, 276 for CYT and 90 for URA were generated. Single point energy calculations on all these complexes were performed at the RI-MP2 level of theory using aug-cc-pVDZ basis set. Since urea molecules are labile in terms of their position and orientation with respect to the 
nucleobases in solution, single point calculations are deemed to be appropriate for sampling these relevant dimer structures. In addition, since we wanted to understand the role of heteroatoms/groups on the stacking interactions, fixing the position/orientation of urea is necessary. Our previous study showed excellent agreement between the results obtained using RI-MP2 and CCSD(T) levels. ${ }^{46}$ The interaction energies were calculated as the difference between the complex energy, and the sum of monomer energies and the basis set superposition errors (BSSE) were corrected using the counterpoise method. Different contributions to the interaction energies were obtained by using the energy decomposition analysis (EDA) method developed by $\mathrm{Su}$ and $\mathrm{Li}$ as implemented in GAMESS. ${ }^{56}$ The RI-MP2 interaction energies were decomposed into electrostatic energy $\left(E_{E S}\right)$, exchange energy $\left(\mathrm{E}_{\mathrm{EX}}\right)$, repulsion energy $\left(\mathrm{E}_{\mathrm{REP}}\right)$, polarization energy $\left(\mathrm{E}_{\mathrm{POL}}\right)$ and dispersion ( $\left.\mathrm{E}_{\mathrm{DIS}}\right)$ energy components.

$\mathrm{E}_{\mathrm{RI}-\mathrm{MP} 2}=\mathrm{E}_{\mathrm{DIS}}+\mathrm{E}_{\mathrm{ES}}+\mathrm{E}_{\mathrm{EX}}+\mathrm{E}_{\mathrm{REP}}+\mathrm{E}_{\mathrm{POL}}$

Natural bond orbital (NBO) ${ }^{57}$ method was used to analyze the interaction of the occupied and unoccupied orbitals with the help of GAMESS. Atoms in molecules (AIM) analysis was performed using the PROAIM program in the AIMALL program package. ${ }^{58}$

\section{Results and Discussion}

\subsection{Interaction energies are highly favorable with respect to the presence of heteroatoms/groups}

Probability distributions of the BSSE corrected interaction energies of all the complexes for each of the four bases are given in Figure 3. All the probability distributions of the energies presented in this manuscript followed the standard histogram analysis with a bin width of $0.5 \mathrm{kcal} / \mathrm{mol}$ and the normalization was done such that the area under the curve is equal to 1 . Interaction energies range from highly favorable to moderately repulsive from about -8 to $4 \mathrm{kcal} / \mathrm{mol}$ for all the nucleobase model systems. This strongly indicates the role of different heteroatoms/groups and orientation of urea molecules with respect to the base model systems on the $\pi$-stacking interactions. It is interesting that with respect to the same number of conjugated $\pi$-electrons on both the nucleobase models and urea, the interaction energies exhibit such a wide range. With respect to the actual base molecule (fully substituted), the interaction energies are highly favorable which indicates the positive contribution of the heteroatoms/groups to the favorable interaction energies (red vertical line in Figure 3). Models which resemble nucleobase structures have favorable energies compared to those which are closer to benzene (with fewer functional groups). A similar analysis was done for each of the models separately and the data corresponding to the guanine base and its model systems are given in Figure 4 and Figures S2-S4 in Supplementary Information. The probability distributions reveal that with respect to the increase in the number of substituents starting from indole (model \# G0), the interaction energies, in general, become more favorable. The order of stability of nucleobase interactions with urea was obtained as GUA $>$ ADE $>$ URA $>$ CYT. Similar to the observation in case of guanine base, the interaction energies in general become more favorable with respect to the inclusion of the heteroatoms/groups in indole/benzene for all systems. For all the systems, complexes containing combinations of amide, carbonyl and amine groups are found to be more stable compared to those that are having only one of these groups. There exist exceptions to this trend indicating that each substituent has different contributions to the overall interaction energy which are analyzed later.

\section{$3.2 \pi$-stacking interactions between urea and nucleobase models are dominated by dispersion-type interactions}

EDA analysis helps to decompose the total interaction energies into different components and gives indications on the nature of these interactions. ${ }^{59,60}$ Even in cases where the total interaction energies are highly stabilizing, the HF component of the energy is observed to be repulsive with positive interaction energies indicating the strong dispersion type interactions in these systems. This is consistent with our previous observation on the complete nucleobase systems. ${ }^{46}$ Interestingly, decomposition of the HF energies indicates that the trends of the interaction energies are dictated by the electrostatic energies. This result is in agreement with previous studies and with the model proposed by Sander et al. 50,61,62 Electrostatic component was attractive for all the cases except for very few cases where the functional group (amide/amine) or the heteroatom of the aromatic system was directly on top of $\mathrm{O} / \mathrm{N}$ of urea causing repulsions. Repulsion component was observed to be always positive with a magnitude in the range of $25-45 \mathrm{kcal} / \mathrm{mol}$ whereas exchange, polarization and dispersion were always attractive in nature. Correlations between different components of the interaction energies (each of them individually, combinations of two and three out of the total of five components) and the total interaction energies of all the 2478 number of model systems were obtained using linear regression analysis. The best linear correlations within each of the three categories are 

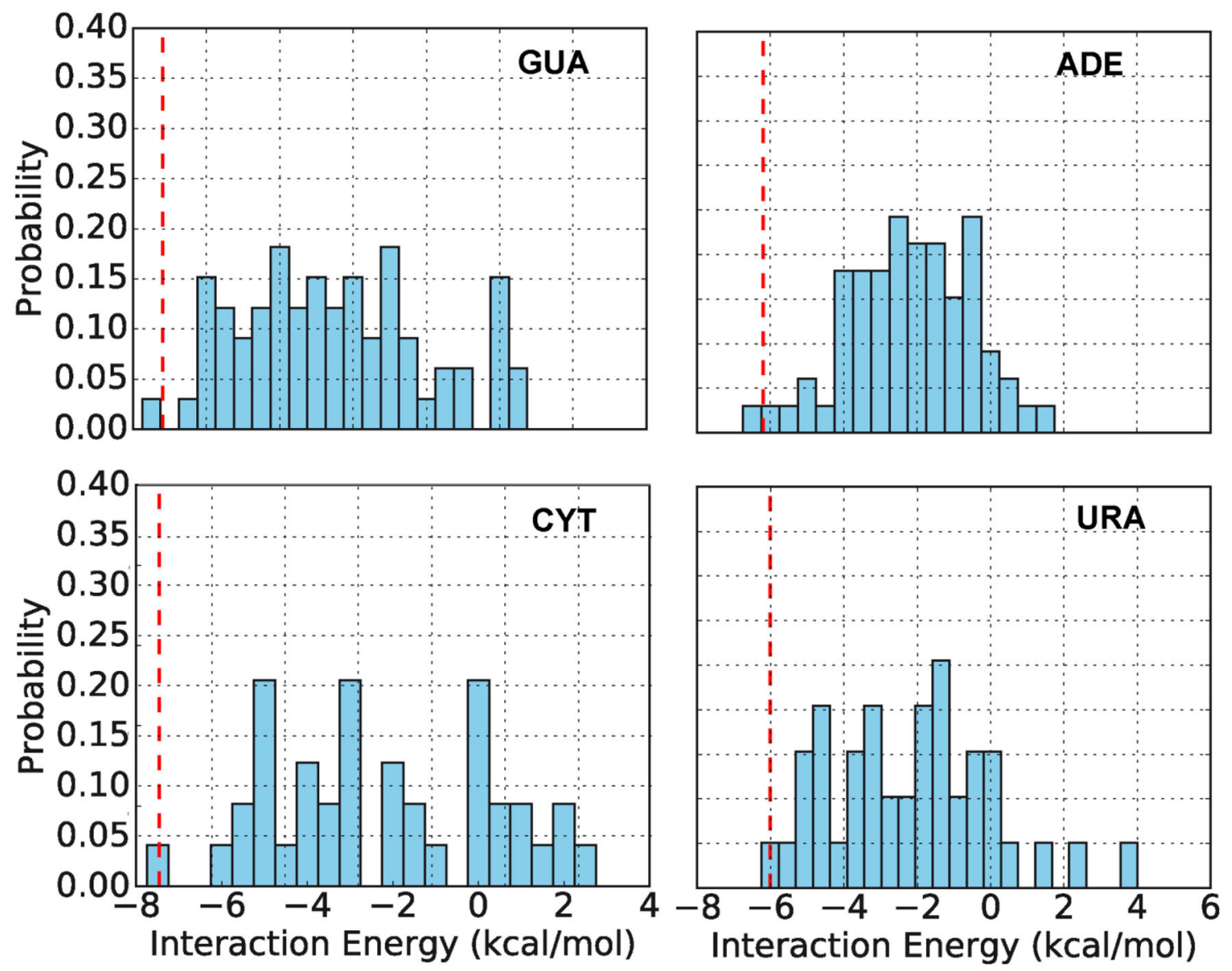

Figure 3. Probability distributions of total interaction energy for GUA, ADE, CYT and URA. Energies for the complexes involving fully substituted models (actual nucleobases) are marked with a red dotted line over the probability plots.

given in Figure 5. Interestingly, though the dispersion energy is responsible for stabilizing the urea-nucleobase $\pi$-stacking interactions, the best correlation between the total interaction energies was observed with the electrostatic energy. Even with the combination of two and three components, best correlations were obtained with combinations of electrostatics, exchange and repulsion components and none of them involved the dispersion energy component of the interaction energy. This further reiterates the above observation that though the stacking interactions are stabilized due to dispersion interactions, the electrostatic effects seem to determine the overall trend of the total interaction energies.

\subsection{Substituent and urea position effects on stabilities of the complexes}

EDA gave the overview of driving forces behind the interactions of aromatic model systems with urea with respect to changing chemical groups. For a better understanding of which of these functional groups has a major impact on stabilizing these complexes with respect to the orientation of urea molecule, further analyses were performed. As mentioned in the methods section, models for nucleobases were generated by changing the following variables to analyze their effects on the strength of interactions: (a) substituents on the $\pi$-system, (b) heteroatoms in the ring and (c) position and orientation of urea with respect to the nucleobase models. Figure 6 gives the interaction energies of guanine model systems with urea with respect to different positions - only the most stable energy among the six possible positions are presented. Plots corresponding to the adenine, cytosine and uracil models are given in Figures S5-S7 in Supplementary Information. Model systems without the amide group ( $3^{\text {rd }}$ variable in case of guanine - Figure 1) seem to have less stability compared to those where this group is present. For example, comparison models G0, G1, G3 and G4, which have no or one substitution, the one with the amide group $(\mathrm{G} 2)$ exhibits favorable interaction energies in general compared to others. This is consistent with adenine, cytosine and uracil as well. Two major 

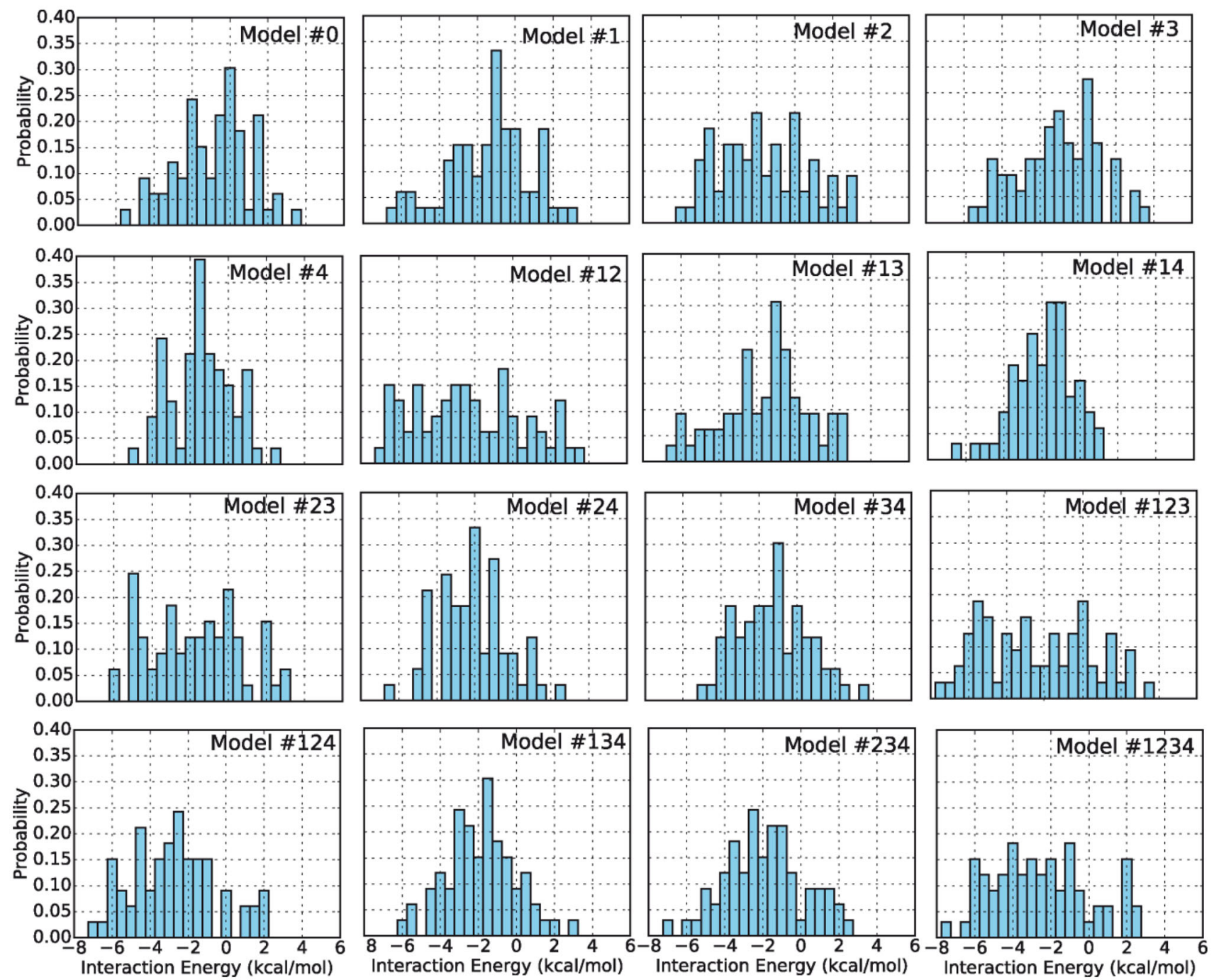

Figure 4. Probability distributions of total interaction energy for each model of guanine system.
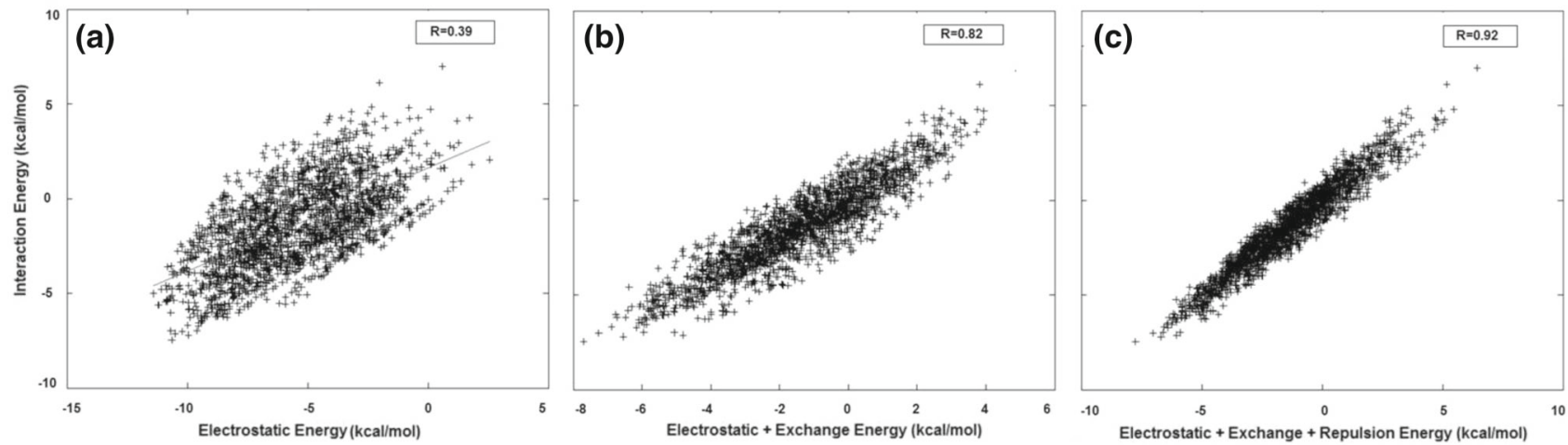

Figure 5. Correlation between total interaction energy and (a) Electrostatics energy (one component), (b) Electrostatic energy and Exchange energy (two components), and (c) Electrostatics energy, Exchange energy and repulsion energy (three components) obtained considering all 2478 models of GUA (1056), ADE (1056), CYT(276), and URA(90), respectively.

components affect the interaction energy trends i.e., heteroatoms depending on the nearby functional group and availability of lone pairs. For example, for GUA models position 9 and 1 were found to be very stabilizing with the majority of the most stable complexes lying on these positions. For models, G0, G1, G3, G4, G13, G14, G34, and G134, i.e., the models lacking amide, the most stable structures were found 


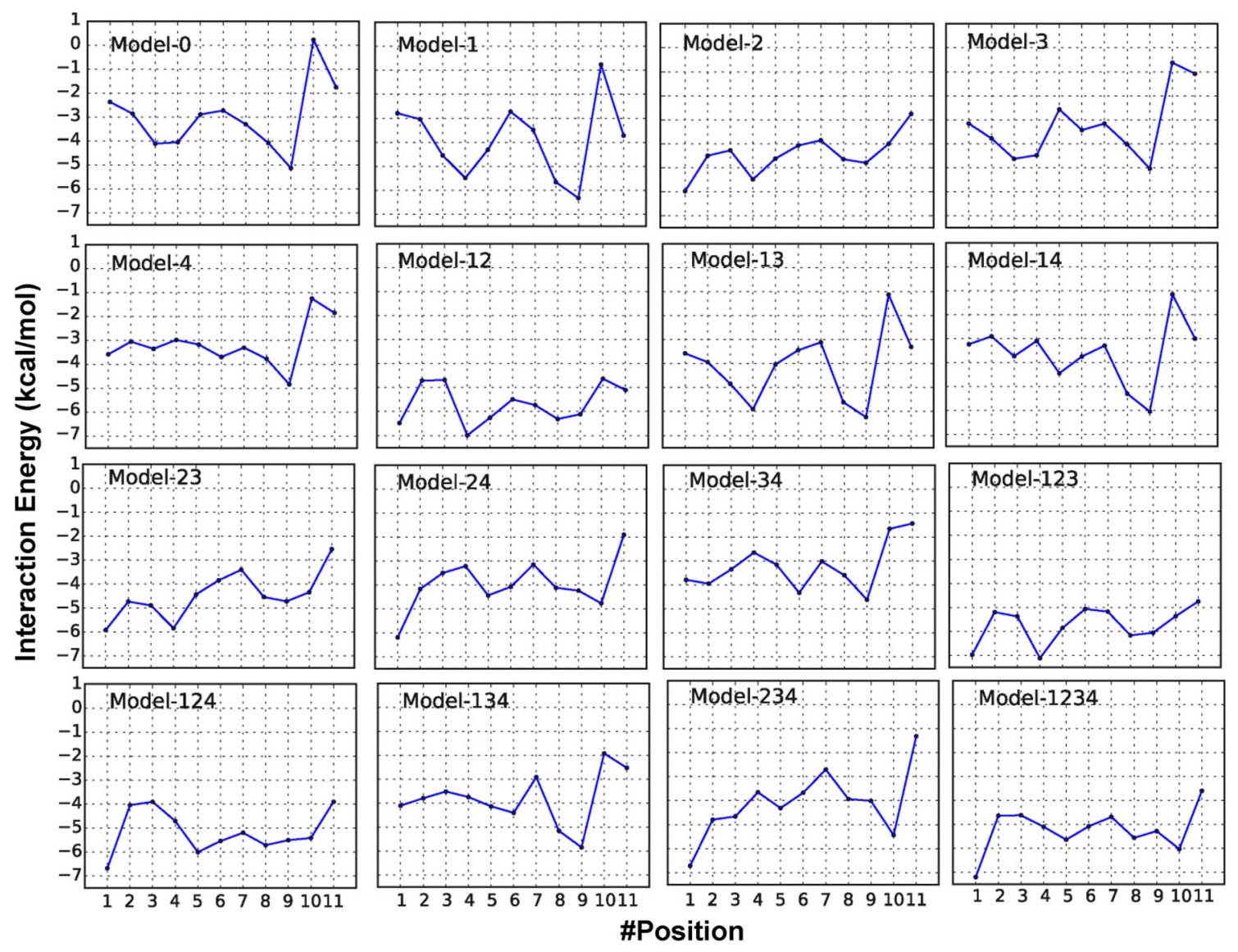

Figure 6. Interaction energy trends for each model of the most stable complexes of the GUA models with respect to 11 positions. All the energies are in $\mathrm{kcal} / \mathrm{mol}$.

to lie on top of position 9-N, whereas, for models, G2, G12, G23, G24, G124, G234 and G1234 (i.e., when amide was introduced), the most stable complex lies on top of position N1. Complexes on the center of the ring were found to be unstable for all the systems that are because of the repulsion factor on those particular positions as can been seen from Figure 7 . With respect to the orientation of urea molecules over model systems, complexes with bond overlap are seen unstable, while complexes on the same position but with different urea orientation becomes stable due to loss of repulsion factor. For all these model systems substituent effects arise due to direct interactions of a carbonyl group on the rings and this supports a hypothesis proposed earlier that substituent effects arise from direct interactions of the substituents with the closest vertex of the other aromatic ring and not from substituent-induced changes in the aryl $\pi$ - system. ${ }^{63-65}$ This quantitative information obtained with this study is helpful to understand the role of functional groups in urea assisted RNA unfolding mechanism. Figure 7 depicts the most stable interaction energy with respect to the model systems of GUA (G0 to G1234) for each of the 11 positions considered here. The corresponding data for the other three bases are presented in Figures S8-S10 of the Supplementary Information. Position 9 where the urea carbon was position directly above the N9 of the nucleobase model system in general was observed to be more stable than the others. However, a zig-zag pattern in general seen in most of these reveals that the stability of stacked complexes depends more on the substituents and less on the position at which the urea is placed with respect to the nucleobase models.

\section{$3.4 N B O$ and AIM analysis}

The nature of the $\pi$-stacking interactions in the model systems was further analyzed using the $\mathrm{NBO}^{56}$ and AIM analysis. ${ }^{58}$ Second-order perturbation theory analysis of the Fock matrix in the NBO basis was used to calculate the stabilizing donor-acceptor interactions between the natural orbitals of urea and nucleobase.$^{57}$ The most stabilizing ones for each nucleobase are given in Figure 8. Orbital interactions are observed from the $\mathrm{C}-\mathrm{C}$ or $\mathrm{C}-\mathrm{N}$ bonding orbitals of the aromatic molecules to the antibonding orbitals of the urea $\mathrm{CO}$ group $(\mathrm{BD}[\mathrm{C}, \mathrm{N}] \mathrm{C} \rightarrow$ $\left.\mathrm{BD}^{*} \mathrm{CO}\right)$. In several systems, the orbital interaction was 

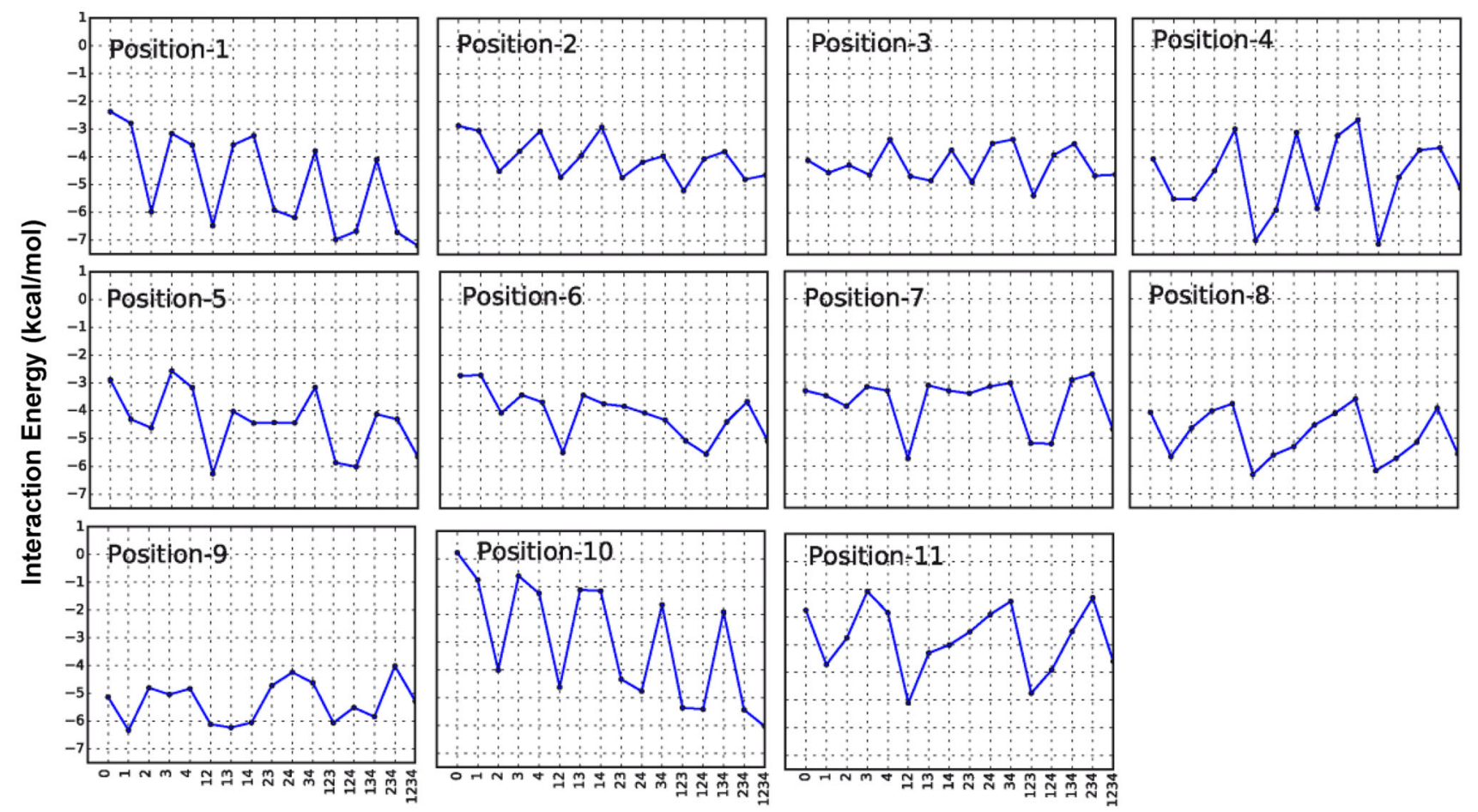

\#Model

Figure 7. Interaction energy trends for each 11 positions separately with respect to all 16 GUA models shown for most stable complexes. All the energies are in $\mathrm{kcal} / \mathrm{mol}$.

established from the aromatic system's lone pair on $\mathrm{N}$ to antibonding orbital of the urea $\mathrm{CO}$ group. It can be seen from Figure 8 that for Guanine system, the nitrogens at position 3 and position 7 did not contribute to orbital interactions with their lone pair, but helped in BD C-N $\rightarrow$ BD* CO type donation, since their lone pair are vested in the aromatic ring, whereas the nitrogen at position 1 and 9 did an $\mathrm{LPN} \rightarrow \mathrm{BD}^{*} \mathrm{CO}$ type of donation leading to complexes on top of these positions to be more stable. The same trend was observed for all other systems where the heteroatom in the ring contributed via $\pi \rightarrow \pi^{*}$ interaction and the nitrogen associated with the amide group contributed via LP $\rightarrow \pi^{*}$ interaction. Most of the stacking complexes had a donation from aromatic system to urea as the most important orbital interaction. However, a donation from urea to aromatic system was also observed. ${ }^{66}$ The lone pair of Nitrogen in urea with interactions like $\mathrm{LPN} \rightarrow \mathrm{BD}^{*} \mathrm{CO}$ ( of aromatic ring) or LP N to an 'empty' lone pair of the urea central $\mathrm{C}$ atom played an important role in donations. This type of lone pair donation between $\mathrm{N}$ and 'empty' $\mathrm{C}$ lone pair has also been observed in several previous studies. ${ }^{67,68}$ In structures where urea was near amide group, the Nitrogen from urea donated back to $\mathrm{CO}$ of amide present in the ring via LP $\rightarrow \pi^{*}$ donation, thus strengthening the $\pi$-stacking interactions. However, the value of these interactions was rather small as compared to the orbital interactions from the aromatic ring to urea. These interactions were also heavily dependent on the orientation of nitrogen atoms of urea and were prominent when $\mathrm{N}$ of urea were on top or near $\pi$ bond of the ring. The $\mathrm{NH}_{2}$ group of aromatic systems did not participate in orbital interactions as no such instance was found for any model of the four systems. Also, on introducing new functional groups from model to model, the original orbital interactions for particular positions remain unperturbed as the $\mathrm{E}(2)$ values for $\pi \rightarrow \pi^{*}$ and other interactions were similar in all the cases. One important thing that has to be noted is that no hydrogen bond like interactions was found. Interactions like $\mathrm{LPN} \rightarrow \mathrm{BD}^{*} \mathrm{NH}$ or $\mathrm{LPO} \rightarrow \mathrm{BD}^{*} \mathrm{NH}$, basically $\pi \rightarrow \sigma$ or LP $\rightarrow \sigma^{*}$ type of interactions were completely absent, even when the $\mathrm{NH}$ of urea were almost on top of $\mathrm{CO}$ of our system. And since, $\mathrm{E}_{\mathrm{HF}}$ is directly correlated with second-order perturbation interactions corresponding to hydrogen bonds, this substantiates our results from EDA where we found the $\mathrm{E}_{\mathrm{HF}}$ to be repulsive in almost all of the cases, whereas the dispersion component of interaction energy was found to be heavily stabilizing as the most prominent interactions that were 

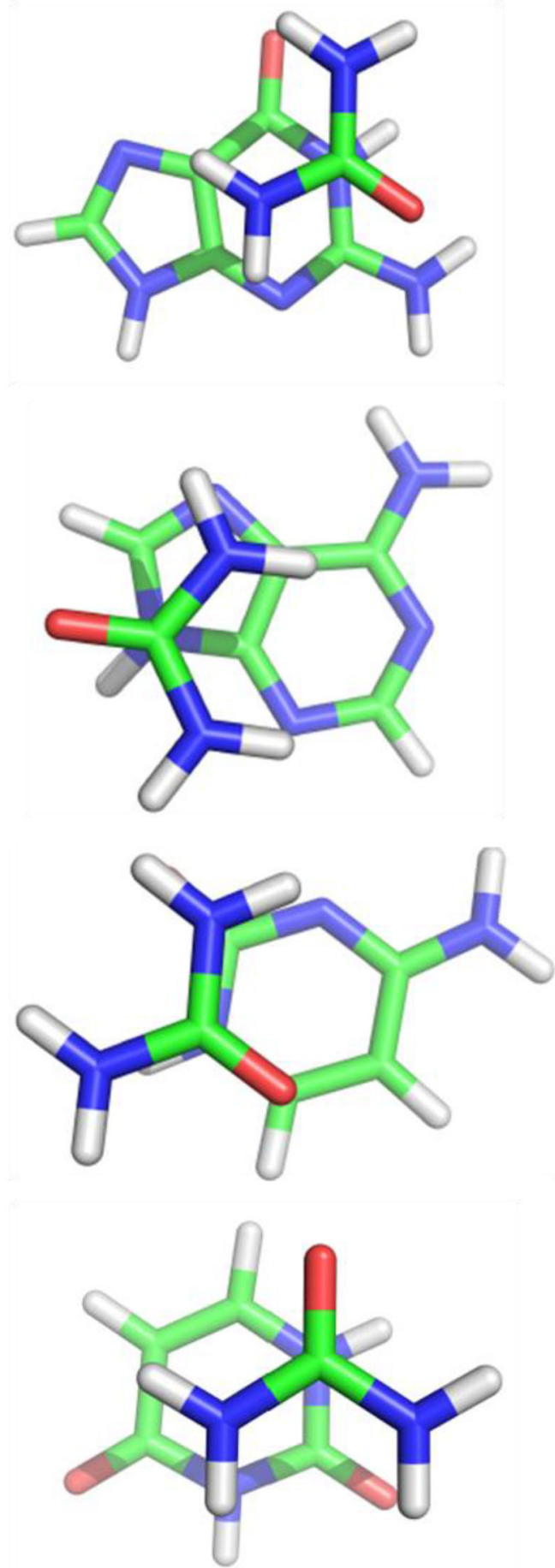

\begin{tabular}{|l|l|}
\hline Orbital Interactions & $\mathrm{E}(2)$ \\
\hline $\mathrm{LPN} 1 \rightarrow \mathrm{BD} * \mathrm{CO}$ & 1.52 \\
$\mathrm{BD}{ }^{*} \mathrm{C} 2-\mathrm{N} \leftarrow \mathrm{BD} C \mathrm{O}$ & 0.40 \\
\hline
\end{tabular}

\section{Guanine}

\begin{tabular}{|l|l|}
\hline Orbital Interactions & $\mathrm{E}(2)$ \\
\hline $\mathrm{BD}$ C8-N9 $\rightarrow \mathrm{BD}^{*} \mathrm{CO}$ & 1.45 \\
$\mathrm{LP}^{*} \mathrm{C} 5 \leftarrow \mathrm{LPN}$ & 1.06 \\
\hline
\end{tabular}

Adenine

\begin{tabular}{|l|l|}
\hline Orbital Interactions & $\mathrm{E}(2)$ \\
\hline $\mathrm{LPN} 3 \rightarrow \mathrm{BD}^{*} \mathrm{CO}$ & 1.89 \\
$\mathrm{BD}^{*} \mathrm{C} 4-\mathrm{C} 5 \leftarrow \mathrm{BD} \mathrm{CO}$ & 0.76 \\
$\mathrm{BD}{ }^{*} \mathrm{C} 2-\mathrm{O} 7 \leftarrow \mathrm{LPN}$ & 1.51 \\
\hline
\end{tabular}

Cytosine

\begin{tabular}{|l|l|}
\hline Orbital Interactions & $\mathrm{E}(2)$ \\
\hline $\mathrm{LPN} 1 \rightarrow \mathrm{BD} \mathrm{D}^{*} \mathrm{CO}$ & 1.52 \\
$\mathrm{BD}{ }^{*} \mathrm{C} 6-\mathrm{C} 5 \leftarrow \mathrm{BD}$ CO & 0.49 \\
$\mathrm{BD}^{*} \mathrm{C} 2-\mathrm{O} \leftarrow \mathrm{LPN}$ & 0.40 \\
$\mathrm{BD}{ }^{*} \mathrm{C} 4-\mathrm{O} \leftarrow \mathrm{LPN}$ & 0.41 \\
\hline
\end{tabular}

\section{Uracil}

Figure 8. The NBO second-order perturbation energy $(\mathrm{E}(2))$ values for the most stable complex of the four nucleobases that were larger than $0.40 \mathrm{kcal} / \mathrm{mol}$ are presented in the figure.

found by NBO correspond to $\pi$-stacking interactions. For each model, AIM analysis was done for the most stable system of that model to get a description of the interactions at play results of which are shown in Figure 9. Several bond critical points and ring critical points corresponding to 4 to 7 membered rings involving atoms of both urea and corresponding complex were observed indicating stabilizing non-covalent interactions between the two molecules in all cases. BCPs were observed between the atoms for which NBO hinted that there might be interactions, thus proving stacking interactions between the two rings in all cases. However, no HB interaction $(\mathrm{N} . . \mathrm{H}$ or $\mathrm{O} \ldots \mathrm{H})$ was found in any system completely ruling out such interactions and making noncovalent interactions the only reasons for the stability of these complexes. The electron density for the BCPS 


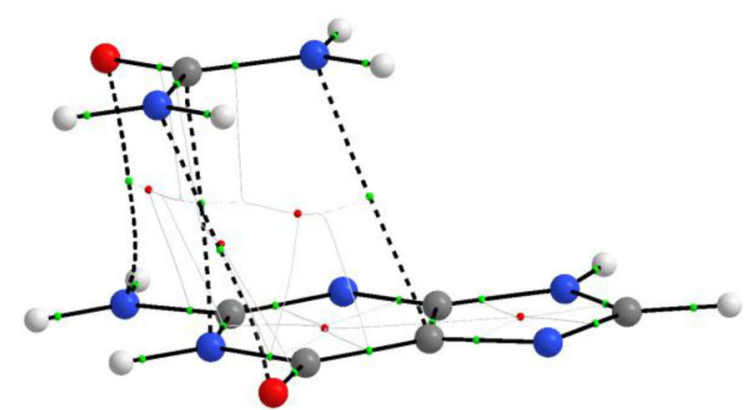

Guanine

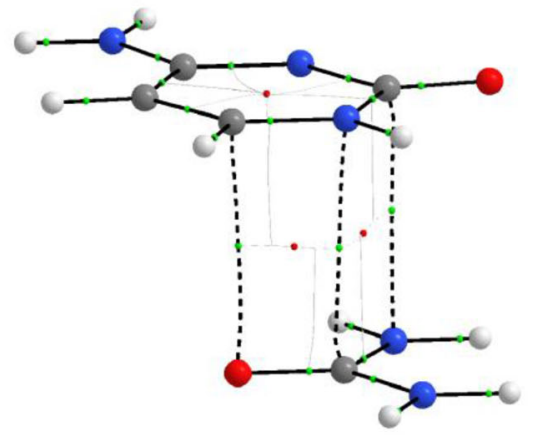

Cytosine

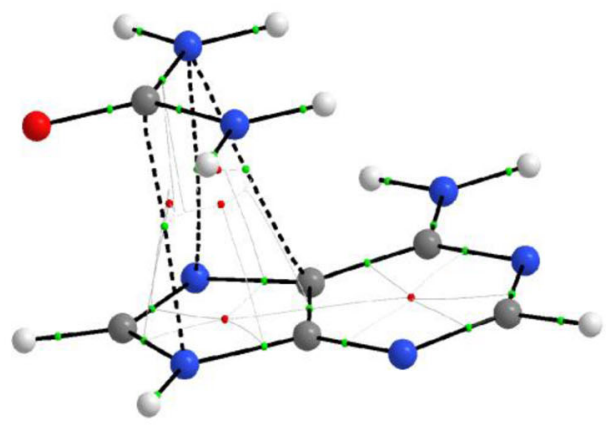

Adenine

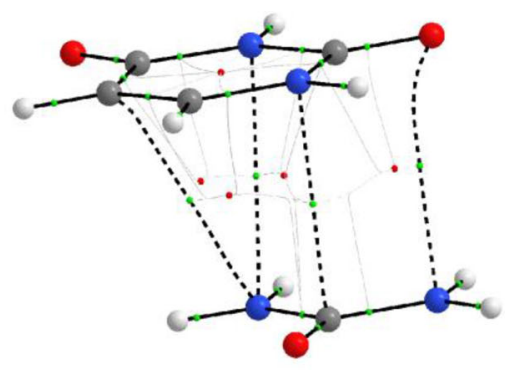

Uracil

Figure 9. The ring critical points (RCP), cage critical points $(\mathrm{CCP})$ and bond critical points $(\mathrm{BCP})$ for the most stable complexes of GUA, ADE, CYT and URA, respectively obtained with AIM analysis. All distances between non-hydrogen atoms that are less than $3 \AA$ are denoted by dotted lines.

found for the non-covalent interactions (N...C, N...O, etc) were of the magnitude of $10^{-3}$ which are much less than the HB type interactions. The Laplacian $\left(\nabla^{2} \rho\right)$ was found to be positive indicating that the interactions are closed-shell interactions.

\section{Conclusions}

The unconventional stacking interactions of urea with RNA nucleobases have been systematically studied using $a b$ initio calculations to understand the role of the heteroatoms of the bases and urea orientation with respect to the aromatic rings. About 2478 model systems were investigated to understand the effect of the substituents, position of urea on the $\pi$-stacking energies. EDA analysis confirmed the dispersion component of the interaction energy to be the most dominant. A strong correlation between total interaction energy and electrostatic component indicates the role of these interactions in determining the trends of the overall stabilities. All the models containing amide and carbonyl groups have favorable interactions with urea compared to other functional groups. Amino $\left(\mathrm{NH}_{2}\right)$ group has a negligible effect on the stability of interactions. The order of stability of bases in interaction with aqueous urea suggests that the chemical nature of nucleic acids is important in understanding the folding and unfolding mechanism and the effect of urea. The results obtained from NBO and AIM analysis explains the nature of non-covalent staking interactions. The NBO analysis showed a considerable amount of $\pi \rightarrow \pi^{*}$ interactions between urea and the base models for the four systems, which corresponds to typical $\pi$ stacking complexes being stabilized by dispersion interactions. The direct interactions between atoms forming stacking interactions obtained in NBO analysis were also observed in AIM analysis supporting the fact that non-covalent stacking interactions are the driving force for ureanucleobase interactions. In conclusion, we propose that heteroatoms, functional groups and orientation of urea molecules are the prominent factors affecting the nature of urea-nucleobase stacking interactions. We conclude that the non-covalent stacking interactions dominated by 
dispersion between exposed surfaces of nucleobases and urea has vital importance in the process of urea driven RNA unfolding. Quantitative information obtained from the functional group analysis performed in this study is helpful in understanding the chemical nature of nucleic acids which affects the folding and unfolding mechanism.

\section{Supplementary Information (SI)}

Variable (functional group) details used for model generation, Interaction energies for ADE, CYT and URA models, with respect to position and with respect to models. Supplementary Information is available at www.ias.ac.in/chemsci.

\section{Acknowledgements}

We thank DST SERB for financial assistance (Grant No. EMR/2016/007697).

\section{References}

1. Mercer T R, Dinger M E and Mattick J S 2009 Long noncoding RNAs: insights into functions Nat. Rev. Genet. 10 155

2. Breaker R R, Gesteland R F, Cech T R and Atkins J F 2006 The RNA world (New York: Cold Spring Harbor Laboratory Press)

3. Blower M D 2013 Molecular insights into intracellular RNA localization Int. Rev. Cell Mol. Biol. 3021

4. Burg M B and Ferraris J D 2008 Intracellular organic osmolytes: function and regulation J. Biol. Chem. 283 7309

5. Yancey P H 2005 Organic osmolytes as compatible, metabolic and counteracting cytoprotectants in high osmolarity and other stresses J. Exp. Biol. 2082819

6. Santoro M M, Liu Y, Khan S M A, Hou L X and Bolen D W 1992 Increased thermal stability of proteins in the presence of naturally occurring osmolytes Biochemistry (Mosc.) 315278

7. Wang A and Bolen D W 1997 A naturally occurring protective system in urea-rich cells: Mechanism of osmolyte protection of proteins against urea denaturation Biochemistry (Mosc.) 369101

8. Schein C H 1990 Solubility as a function of protein structure and solvent components Nat. Biotechnol. 8308

9. Zhou R, Li J, Hua L, Yang Z and Berne B J 2011 Comment on "urea-mediated protein denaturation: A consensus view" J. Phys. Chem. B 1151323

10. Li W, Zhou R and Mu Y 2012 Salting effects on protein components in aqueous $\mathrm{NaCl}$ and urea solutions: toward understanding of urea-induced protein denaturation $J$. Phys. Chem. B 1161446

11. Wallqvist A, Covell D G and Thirumalai D 1998 Hydrophobic interactions in aqueous urea solutions with implications for the mechanism of protein denaturation J. Am. Chem. Soc. 120427

12. Guinn E J, Pegram L M, Capp M W, Pollock M N and Record M T 2011 Quantifying why urea is a protein denaturant, whereas glycine betaine is a protein stabilizer Proc. Natl. Acad. Sci. 10816932

13. Hua L, Zhou R, Thirumalai D and Berne B J 2008 Urea denaturation by stronger dispersion interactions with proteins than water implies a 2-stage unfolding Proc. Natl. Acad. Sci. 10516928

14. Lambert D and Draper D E 2012 Denaturation of RNA secondary and tertiary structure by urea: simple unfolded state models and free energy parameters account for measured m-values Biochemistry (Mosc.) 51 9014

15. Priyakumar U D, Hyeon C, Thirumalai D and MacKerell Jr A D 2009 Urea destabilizes RNA by forming stacking interactions and multiple hydrogen bonds with nucleic acid bases J. Am. Chem. Soc. 13117759

16. Shelton V M, Sosnick T R and Pan T 1999 Applicability of urea in the thermodynamic analysis of secondary and tertiary RNA folding Biochemistry (Mosc.) 3816831

17. Canchi D R, Paschek D and García A E 2010 Equilibrium study of protein denaturation by urea J. Am. Chem. Soc. 1322338

18. Stumpe M C and Grubmüller H 2007 Interaction of urea with amino acids: implications for urea-induced protein denaturation J. Am. Chem. Soc. 12916126

19. Guinn E J, Schwinefus J J, Cha H K, McDevitt J L, Merker W E, Ritzer R, Muth G W, Engelsgjerd S W, Mangold K E and Thompson P J 2013 Quantifying functional group interactions that determine urea effects on nucleic acid helix formation J. Am. Chem. Soc. 135 5828

20. Ma L, Pegram L, Record Jr M T and Cui Q 2010 Preferential interactions between small solutes and the protein backbone: A computational analysis Biochemistry (Mosc.) 491954

21. Auton M and Bolen D W 2005 Predicting the energetics of osmolyte-induced protein folding/unfolding Proc. Natl. Acad. Sci. U. S. A. 10215065

22. Baldwin R L 1975 Intermediates in protein folding reactions and the mechanism of protein folding Annu. Rev. Biochem. 44453

23. Makhatadze G I and Privalov P L 1992 Protein interactions with urea and guanidinium chloride: a calorimetric study J. Mol. Biol. 226491

24. Goyal S, Chattopadhyay A, Kasavajhala K and Priyakumar U D 2017 Role of Urea-Aromatic Stacking Interactions in Stabilizing the Aromatic Residues of the Protein in Urea-Induced Denatured State J. Am. Chem. Soc. 139 14931

25. Schneck E, Horinek D and Netz R R 2013 Insight into the molecular mechanisms of protein stabilizing osmolytes from global force-field variations J. Phys. Chem. B 117 8310

26. Bennion B J and Daggett V 2003 The molecular basis for the chemical denaturation of proteins by urea Proc. Natl. Acad. Sci. 1005142

27. Levy Y and Onuchic J N 2006 Water mediation in protein folding and molecular recognition Annu. Rev. Biophys. Biomol. Struct. 35389

28. Vanzi F, Madan B and Sharp K 1998 Effect of the protein denaturants urea and guanidinium on water structure: A structural and thermodynamic study J. Am. Chem. Soc. 12010748 
29. Berteotti A, Barducci A and Parrinello M 2011 Effect of Urea on the $\beta$-Hairpin Conformational Ensemble and Protein Denaturation Mechanism J. Am. Chem. Soc. 133 17200

30. Stumpe M C and Grubmüller H 2007 Aqueous urea solutions: structure, energetics, and urea aggregation J. Phys. Chem. B 1116220

31. Guinn E J, Pegram L M, Capp M W, Pollock M N and Record M T 2011 Quantifying why urea is a protein denaturant, whereas glycine betaine is a protein stabilizer Proc. Natl. Acad. Sci.

32. Liu C, Wang T, Bai Y and Wang J 2017 Electrostatic forces govern the binding mechanism of intrinsically disordered histone chaperones PLOS ONE 12 e0178405

33. Rocco A G, Mollica L, Ricchiuto P, Baptista A M, Gianazza E and Eberini I 2008 Characterization of the protein unfolding processes induced by urea and temperature Biophys. J. 942241

34. Sánchez I E and Kiefhaber T 2003 Evidence for sequential barriers and obligatory intermediates in apparent two-state protein folding J. Mol. Biol. 325367

35. Streicher W W and Makhatadze G I 2007 Unfolding thermodynamics of Trp-cage, a 20 residue miniprotein, studied by differential scanning calorimetry and circular dichroism spectroscopy Biochemistry (Mosc.) 46 2876

36. England J L and Haran G 2011 Role of solvation effects in protein denaturation: from thermodynamics to single molecules and back Annu. Rev. Phys. Chem. 62 257

37. Miner J C and García A E 2017 Equilibrium Denaturation and Preferential Interactions of an RNA Tetraloop with Urea J. Phys. Chem. B 1213734

38. Herschlag D 1995 RNA chaperones and the RNA folding problem J. Biol. Chem. 27020871

39. Kiger A A, Baum B, Jones S, Jones M R, Coulson A, Echeverri C and Perrimon N 2003 A functional genomic analysis of cell morphology using RNA interference $J$. Biol. 227

40. Mercer T R and Mattick J S 2013 Structure and function of long noncoding RNAs in epigenetic regulation Nat. Struct. Mol. Biol. 20300

41. McKay D B 1996 Structure and function of the hammerhead ribozyme: an unfinished story RNA 2395

42. Wada A and Suyama A 1986 Local stability of DNA and RNA secondary structure and its relation to biological functions Prog. Biophys. Mol. Biol. 47113

43. Lambert D and Draper D E 2007 Effects of osmolytes on RNA secondary and tertiary structure stabilities and RNA-Mg2+ interactions J. Mol. Biol. 370993

44. Pincus D L, Hyeon C and Thirumalai D 2008 Effects of trimethylamine N-oxide TMAO and crowding agents on the stability of RNA hairpins J. Am. Chem. Soc. 130 7364

45. Yoon J, Thirumalai D and Hyeon C 2013 Urea-induced denaturation of preQ1-riboswitch J. Am. Chem. Soc. 135 12112

46. Kasavajhala K, Bikkina S, Patil I, MacKerell Jr A D and Priyakumar U D 2015 Dispersion interactions between urea and nucleobases contribute to the destabilization of RNA by urea in aqueous solution J. Phys. Chem. B 119 3755
47. Salonen L M, Ellermann M and Diederich F 2011 Aromatic rings in chemical and biological recognition: energetics and structures Angew. Chem. Int. Ed. 50 4808

48. Schottel B L, Chifotides H T and Dunbar K R 2008 Anion- $\pi$ interactions Chem. Soc. Rev. 3768

49. Gallivan J P and Dougherty D A 1999 Cation- $\pi$ interactions in structural biology Proc. Natl. Acad. Sci. 96 9459

50. Hunter C A, Lawson K R, Perkins J and Urch C J 2001 Aromatic interactions J. Chem. Soc. Perkin Trans. 2 651

51. Knowles R R and Jacobsen E N 2010 Attractive noncovalent interactions in asymmetric catalysis: links between enzymes and small molecule catalysts Proc. Natl. Acad. Sci. 201006402

52. Zürcher M and Diederich F 2008 Structure-based drug design: exploring the proper filling of apolar pockets at enzyme active sites J. Org. Chem. 73 4345

53. Schneider H-J 2009 Binding mechanisms in supramolecular complexes Angew. Chem. Int. Ed. 483924

54. Weigend F, Häser M, Patzelt H and Ahlrichs R 1998 RIMP2: optimized auxiliary basis sets and demonstration of efficiency Chem. Phys. Lett. 294143

55. Schmidt M W, Baldridge K K, Boatz J A, Elbert S T, Gordon M S, Jensen J H, Koseki S, Matsunaga N, Nguyen K A and Su S 1993 General atomic and molecular electronic structure system J. Comput. Chem. 14 1347

56. Su P and Li H 2009 Energy decomposition analysis of covalent bonds and intermolecular interactions J. Chem. Phys. 131014102

57. Reed A E, Curtiss L A and Weinhold F 1988 Intermolecular interactions from a natural bond orbital, donor-acceptor viewpoint Chem. Rev. 88899

58. Todd A and Keith T K 2011 AIMALL Version 11.12. 19. Overland Park KS, USA: Gristmill Software

59. Kitaura K and Morokuma K 1976 A new energy decomposition scheme for molecular interactions within the Hartree-Fock approximation Int. J. Quantum Chem. 10 325

60. Glendening E D and Streitwieser A 1994 Natural energy decomposition analysis: An energy partitioning procedure for molecular interactions with application to weak hydrogen bonding, strong ionic, and moderate donor-acceptor interactions J. Chem. Phys. 100 2900

61. Lee E C, Kim D, Jurečka P, Tarakeshwar P, Hobza P and Kim K S 2007 Understanding of assembly phenomena by aromatic- aromatic interactions: benzene dimer and the substituted systems J. Phys. Chem. A 111 3446

62. Hunter C A and Sanders J K 1990 The nature of. pi.-. pi. interactions J. Am. Chem. Soc. 1125525

63. Wheeler S E 2011 Local nature of substituent effects in stacking interactions J. Am. Chem. Soc. 13310262

64. Wheeler S E 2012 Understanding substituent effects in noncovalent interactions involving aromatic rings Acc. Chem. Res. 461029

65. Wheeler S E and Houk K N 2008 Substituent Effects in the Benzene Dimer are Due to Direct Interactions of 
the Substituents with the Unsubstituted Benzene J. Am. Chem. Soc. 13010854

66. Cheng X, Shkel I A, O’Connor K, Henrich J, Molzahn C, Lambert D and Record Jr M T 2017 Experimental Atom-by-Atom Dissection of Amide-Amide and Amide-Hydrocarbon Interactions in $\mathrm{H}_{2} \mathrm{O} \mathrm{J}$. Am. Chem. Soc. 1399885
67. Blanco F, Kelly B, Sánchez-Sanz G, Trujillo C, Alkorta I, Elguero J and Rozas I 2013 Non-covalent interactions: complexes of guanidinium with DNA and RNA nucleobases J. Phys. Chem. B 11711608

68. Blanco F, Kelly B, Alkorta I, Rozas I and Elguero J 2011 Cation $-\pi$ interactions: Complexes of guanidinium and simple aromatic systems Chem. Phys. Lett. 511129 